\title{
Congestion Control Call Admission Control (CC-CAC) Algorithm for Mobile Broadband Networks
}

\author{
Ibrahim S., Abubakar R., Nasir A.S., and Solomon Y.
}

\begin{abstract}
Mobile broadband networks are continuously faced with the challenge of limited resources due to the increasing demand for high speed Internet services. To manage these resources, call admission control (CAC) which determines whether or not a connection request should be accepted or rejected is one of the resource management techniques usually deployed. A Dynamic QoS-aware CAC algorithm was recently proposed to improve resource utilization. However, the algorithm detects congestion of the network only after it has occurred, which leads to increase in blocking probability and thus results to reduction in throughput. In this paper, a congestion control call admission control (CC-CAC) algorithm is proposed to reduce call blocking and increase throughput of all connections. The $\mathrm{CC}$ CAC employs a congestion control mechanism that regulates the base station buffer to operate at a target threshold. It also employs a dynamic bandwidth degradation mechanism to degrade the resources of the admitted connections when resources are insufficient to admit more connections. The algorithm is evaluated using simulation. The results show that the proposed scheme outperforms the benchmark scheme in terms of decrease in blocking probability and increase in throughput for all connection classes.
\end{abstract}

Index Terms-Quality of service; Wireless Metropolitan Network; WiMAX; CAC algorithm; and bandwidth degradation

\section{INTRODUCTION}

The proliferations of mobile devices such as mobile phones, smart phones, and tablets and advancement in wireless technologies have led to increase in demand for Internet services such as voice over Internet protocol (VoIP), video streaming, internet surfing, and online gaming etc. by users, anywhere and anytime. Due to quality of service (QoS) requirement constraints of these services such as delay, jitter,throughput and packet loss, therefore mobile broadband technologies become necessary such as Long-Term Evolution (LTE) network and Worldwide Interoperability for Microwave Access (WiMAX) becomes necessary [1] [2].

WiMAX is a technology rolled out by the IEEE working group. It is usually referred to as the 802.16 standard designed to achieve higher data-rate, lower latency, improved system capacity as well as extended coverage [3]. The technology deploys a set of radio resources management techniques to effectively manage the network resources [4] in order to achieve the QoS requirements. These techniques consist of scheduling and call admission control (CAC). CAC admits or

I.S. is with the Department of Information and Communication Technology, Usmanu Danfodiyo University Sokoto (UDUS), Nigeria (email: Ibrahim.saidu@udusok.edu.ng).

A. R. is with the Department of Computer Science, UDUS, Nigeria (email: abroko@lyahoo.com). rejects a new connection request if the required QoS of the new connection request will be met without lowering that of the ongoing calls [5].

Several research works on CAC have been conducted for QoS provisioning $[3,4,5,6]$. The schemes proposed in $[3,4]$ efficiently utilize resources but the admission criteria deployed starve the high and low priority connections under moderate to heavy traffic load. The schemes also waste resources when the handoff connection (HC) traffic is below the anticipated level due to a fixed reservation technique used. While the scheme in [5] enhances resource utilization and guarantees QoS for all classes. Furthermore, the existing scheme in [6], which is an improvement to [3] deploys a bandwidth pre-check mechanism to determine whether or not resources obtained from the degradation procedure will be sufficient to meet the bandwidth demands of the requesting connection before the degradation is performed, thus determining the level of tolerable congestion in the network. Despite its performance, the scheme increases call blocking rates: hence decreases throughput because it only detects congestion after it has occurred.

In this paper, a congestion control call admission control (CC-CAC) algorithm is proposed to minimize congestion. The algorithm deploys a congestion control mechanism to determine the level of congestion in the network at every point. It also uses a dynamic bandwidth degradation mechanism to admit more connections into the network when resources are inadequate. The performance of the proposed algorithm against the DQA-CAC algorithm in [3] is assessed using a discrete event simulator. The simulation results indicate that the proposed CC-CAC surpasses the compared scheme in terms of decrease in blocking probability and increase in throughput for all connection classes.

The rest of the paper is organized as follows. Related works on call admission control algorithms in Section II. The basic idea of Dynamic QoS-Aware CAC algorithm is presented in Section III. The performance comparison is discussed in Section IV. Finally, Section V concludes the paper.

\section{RELATED WORKS ON CAC SCHMES}

This section presents a review on several CAC schemes that have been proposed to ensure efficient management of wireless network resources as well as QoS as follows:

N.A.S. is with the Directorate of ICT, Umar Musa Yar'adua University, Katsina, Nigeria (e-mail: nashinkafi@gmail.com).

S.Y. is with the with the Department of Computer Science, UDUS,, , Nigeria (e-mail: yese@gmail.com). 
In [1], a dynamic Call Admission Control (CAC) and Resource Reservation (RR) scheme is proposed to ensure service differentiation. The scheme uses guard channel mechanism to select resources according to the estimated number of incoming call request of various service classes. It also employs a dynamic resource reservation algorithm to efficiently estimate resources needed to be reserved for high priority calls by using signal to noise (SNR) and information of mobile users in neighboring cells. The proposed scheme reduces handoff dropping probability but it is unfair to lower priority calls and wastes resources due to the static reservation policy used.

In [7], an efficient call admission control scheme is proposed to ensure QoS. The scheme employs the concept of adaptive bandwidth degradation to obtain the minimum required bandwidth for handoff and new connections. The scheme gives equal priority to all handoff connections and adaptively degrades both rtPS and nrtPS connections. The scheme maximizes bandwidth utilization. The adaptive degradation has a minor effect on the dropping and blocking probability of all service class. However, the arrival rate and connection rate is assumed to be the same which is unrealistic. Also, the non-prioritization of handoff connections may lead to increased dropping probability of handoff connections which are more critical to the network.

In [3], an efficient admission control scheme is proposed to reduce handoff service flow dropping probability. The proposed scheme degrades the bandwidth of the active service flow when there is no available bandwidth to admit a handoff connection during heavy network load condition, thereby decreasing the handoff service dropping probability and the degraded bandwidth will be upgraded in reverse order when the network load reduces. The scheme improves resource utilization due to the bandwidth upgrading procedure and reduces the HCDP but is scheme unfair to new connections and can increase their blocking rates because the stepwise degradation of lower priority is to only admit handoff connections.

An efficient Call Admission Control (CAC) scheme is proposed in [2] to satisfy both bandwidth and delay guarantee to the admitted connections. The scheme deploys a token bucket mechanism that guarantees bandwidth to all service classes and delay guarantees to delay sensitive rtPS connections. The scheme checks the availability of resources and the delay requirement of existing rtPS connections to admit a new connection into the network; otherwise the new connection is rejected. The scheme also considered ertPS and rtPS connections to be the same because both connections have same QoS parameters and differ only by the way of request/transmission. Furthermore, it uses a stepwise degradation policy to degrade rtPS and nrtPS connections in order to accept new connections into the network. The scheme reduces new $\mathrm{CBP}$ and handoff CDP of UGS and rtPS. However, the scheme uses stepwise degradation policy, which may result to waste of resources and increased delay because the bandwidth of rtPS and nrtPS connections may be degraded more than required.

To provide QoS to all service classes, a new Call Admission Control Algorithm (CAC) proposed in [8]. The proposed scheme admits new request for some certain time slot temporarily. The scheme check each time slot to accept high priority connections if there is enough resources otherwise it is rejected. The scheme decreases the departure rate of ongoing connections and increases the departure rate for new connections. The scheme gives handoff and UGS connections highest priority. The scheme reduces the blocking probability of new calls and dropping probability of handoff call thereby providing good quality of service for HCs and NCs. However, the scheme increases the delay of existing real time connections as a result of the increase in departure rate of new connections.

A packet scheduling CAC scheme is proposed in [9] to provide QoS. The scheme deploys a traffic policing and admission control module. The traffic policing module prevents the higher priority connections from using bandwidth more than their allocated bandwidth and also monitors violation of QoS contracts by admitted connection. The scheme reserves bandwidth for UGS and rtPS connections while the remainder is used for other service classes. It provides bandwidth guarantees to real time service classes and ensures a delay guarantee for rtPS when the bandwidth requirements are satisfied. However, the scheme starves low priority service class and ignores consideration of handoff connections which can lead to increased handoff call dropping if deployed for real networks.

In [10], a dynamic admission control scheme is proposed to ensure QoS for UGS connections. The scheme employs bandwidth reservation and degradation policy to provide QoS for different service class. The proposed scheme degrades nrtPS connection in a stepwise manner when resources are insufficient in order to admit new calls into the network. The scheme gives maximum priority to UGS service class and reduces Call Blocking Probability (CBP) for UGS. However, it increases the CBP of nrtPS due to the degradation policy used.

In [11], an enhanced call admission control scheme is proposed to mitigate the unfair treatment of non-UGS connections. The scheme uses a bandwidth reservation technique to reserve bandwidth for non-UGS service classes when VOIP call traffic increases above threshold. The scheme decreases the available bandwidth for the connection of rtPS and nrtPS service class using the reservation technique. The scheme decreases the blocking probability of UGS because a portion of bandwidth is dedicated to UGS service class. However, there may be wastage of resource if the reserved bandwidth is not fully utilized.

To improve resource utilization, a Modulation aware CAC scheme is proposed in [12]. The proposed scheme considers maximum sustainable traffic rate (MSTR) as an admission criteria to accept new UGS, ertPS and rtPS connections and average rate for nrtPS connections when the network is less congested but when the network is congested, admission criterion for rtPS is average rate and minimum required traffic rate (MRTR) for nrtPS connections. The scheme improves on the admission of higher priority real time service class by degrading the bandwidth of the admitted lower priority connections. However, the scheme ignores the consideration of handoff connections which may lead to increase in call dropping probability.

In [13], a dynamic connection admission control (CAC) and bandwidth reservation (BR) scheme is proposed. The proposed scheme adjusts the admission of criteria on call depending on the network loads and uses an adaptive QoS provisioning strategy to enhance the efficiency of bandwidth utilization. The scheme adjusts the amount of reserved bandwidth for handoff calls according to the arrival rate distribution of both handoff and new connections. The scheme decrease blocking probability of UGS service class. However, the blocking probability of rtPS and nrtPS increases due to the reservation policy used. 
In [14], an improved CAC scheme with adaptive bandwidth reservation and degradation policy is proposed to prevent call blocking. The proposed scheme incorporates bandwidth reservation policy to reserve fix amount of resource for handoff calls and dynamic amount for new calls based on the arrival of $\mathrm{HC}$ and NC traffic. The bandwidth degradation policy uses a non-linear technique to degrade the bandwidth of the admitted service classes according to traffic load under insufficient resources. The scheme reduces the CBP through complete allocation of bandwidth to new calls. However, the scheme uses fixed reservation technique for HCs which may waste network resources when $\mathrm{HC}$ traffic is low.

In [3], a QoS Aware CAC with bandwidth reservation and bandwidth degradation for 802.16 networks to enhance utilization of network resources and prevent starvation of service classes. The scheme determines the admission of calls based on scheduling of service class in order to prevent starvation of service classes. The scheme uses bandwidth degradation to admit more users when there is no available bandwidth to admit a user. The scheme uses adaptive threshold to decide the quantity of reserved bandwidth for handoff connections based on traffic intensity of handoff connections. The scheme also uses adaptive threshold that is dynamically changed based on arrival rate of new or handoff connections. The scheme accommodates more users into the network and guarantees QoS to service classes. However, it wastes resources due to fixed BD policy used.

In [6], a Dynamic QoS-Aware Call Admission Control Algorithm (DQA-CAC) was proposed to improve resources utilization. The scheme deploys a bandwidth pre-check mechanism to determine whether or not the resources acquired from degradation can sufficiently meet the bandwidth required by a requesting connection before the degradation is performed. The scheme also deploys a dynamic degradation mechanism that degrades variable amount of resources from existing ertPS and rtPS connections when available resources are insufficient to accept a connection request. The scheme improves resource utilization as it lowers new call blocking rate. However, the scheme also underutilizes network resources and reduces the system throughput because it only detects congestion after the congestion has occurred.

To fix the shortcoming highlighted above, we propose a Dynamic QoS-Aware CAC algorithm with co congestion control to prevent resource wastage and increase overall system throughput.

\section{PROPOSED CC-CAC}

This section presents the CC-CAC algorithm, a variant of the DQA-CAC algorithm presented in [6]. However, the drawback of the DQA-CAC algorithm is highlighted. The algorithm admits high priority connections based on their maximum sustainable traffic rate (MSTR) and admits low priority connections according to their minimum required traffic rate (MRTR). The scheme degrades rtPS and ertPS calls when resources are insufficient, due to their flexible QoS requirements so as to admit more connections into the network. The scheme deploys a pre-check mechanism to determine the sufficiency or otherwise of the resources to be acquired from the degradation procedure in meeting the demand of the requesting connection without overcongesting the network and violating the QoS of existing connections. However, the algorithm only detects congestion after it has occurred, leading to dropping of calls and consequently reduced throughput.

To address the problem highlighted above, a CC-CAC Algorithm is proposed. First, the scheme adopts an adaptive bandwidth reservation threshold in [3] to reserve resources for handoff calls.

First, it deploys a congestion control mechanism to determine the level of congestion in the network. The mechanism is derived as follows:

Firstly, the system's current bandwidth utilization is presented as

$B_{U}=\sum_{i=1}^{n}\left(B_{U G S}+B_{\text {ertPS }}+B_{r t P S}+B_{n r t P S}+B_{B E}\right)$

Where $B_{U G S}, B_{\text {ertPS }}, B_{r t P S}, B_{n r t P S}$ and $B_{B E}$ corresponding to the bandwidth in use by UGS, ertPS, rtPS, nrtPS and BE connections respectively.

Then, the congestion control mechanism is introduced to regulate to indicate the amount of congestion that the base station (BS) can tolerate. The mechanism is as follows:

$f(q)=\left\{\begin{array}{c}\frac{B_{l}-q_{l}}{B_{l}-q_{0}}, \text { if } q_{0} \leq q_{l} \\ \frac{(\alpha-1) *\left(q_{0}-q_{l}\right)}{q_{0}+1}, \text { if } q_{0} \geq q_{l} \\ 1, \text { if } q_{l}=0\end{array}\right.$

Where $B_{l}$ is the buffer length at the BS and $\alpha$ is underutilization factor, $q_{l}$ is the queue length, $q_{0}$ is the threshold operating point.

The threshold operating point is computed as

$$
q_{0}=B_{l} * B_{u}
$$

Where $B_{u}$ is the buffer utilization.

Next, the uses Explicit Rate $\left(E_{r}\right)$ to determine how much traffic to be handled by the BS. The $\left(E_{r}\right.$ is sent as a feed back to the MSs which is derived as

$E_{r}=M_{r} * f(q)$

The expected rate for each class $i$

$E_{r}=M_{r}(t-1) * f(q)$

To meet the QoS requirements of each $i$, the scheme divides the MRTR and MSTR per second into the MRTR and MSTR per frame using Equations (6) and (7).

$$
\begin{aligned}
& M R T R=F_{i}\left(M_{R T R} * F_{d}\right) \\
& M S T R=F_{i}\left(M S T R_{s} * F_{d}\right)
\end{aligned}
$$

The congestion control component of the call admission control scheme checks to ascertain if the bandwidth requested 
by the connection can be met without overshooting the set congestion threshold. That is, if in Equation (2), $f(q)>1$, it indicates network underutilization. If the value of $f(q)<1$, it shows network congestion. At this point, the degradation mechanism of the CAC scheme is triggered to degrade resources from existing connections in order to admit the connection request, starting with the lowest priority connections.

The degradation step size of the degradation procedure is calculated as

First, the current bandwidth utilization after degradation before assignment is given as

$$
B_{u}^{d}=B_{U}-B_{n}^{d}
$$

where $B_{n}^{d}$ is the amount of bandwidth needed from the degradation procedure which is derived as,

$B_{n}^{d}=B_{r}-B_{a} \quad$ for every $B_{r}>B_{a}$

where $B_{a}$ is the bandwidth available in the system.

Then, the bandwidth intelligent function $(I(B))$ is given as:

$I(B)=1-\frac{B_{t}-B_{u}}{B_{t}-B_{n}^{d}}$

Finally, the variable step size is calculated as follows:

$\ell=I(B) * B_{d, i}$

where $B_{d, i}$ is the allowed bandwidth to be assigned after degradation which is MSTR for UGS and MRTR for other class of connections. The admission of UGS connections based on their MSTR requirement and others on MRTR is because UGS connections require constant bit rates while the other classes have adjustable QoS requirements.

\section{PERFROMANCE EVALUATION}

The performance evaluation of the proposed CAC scheme against the QACAC-BR-BD in [3] is implemented on a discrete event simulator developed using $\mathrm{C}++$ programming language. The evaluation is based on the $\mathrm{NC}$ blocking rate and the $\mathrm{HC}$ dropping rates. The simulation captures the new connection and handoff connection scenarios. The arrival of the connections is assumed to follow a Poisson distribution. The average number of connections leaving the system is assumed to be 1/10th of the number entering, with the base station knowing the bandwidth requirements of each connection with respect to its current modulation and coding scheme. Also, other simulation parameters are: $10 \mathrm{MHz}$ bandwidth, 1024 FFT size, with 184, 120 and 720 null, pilot and Data subcarriers respectively. Furthermore, a symbol period of $102.9 \mu \mathrm{s}, 5 \mathrm{~ms}$ frame duration, 48 OFDM and 44 Data OFDM symbols are used. The simulation topology is shown in Fig.1.

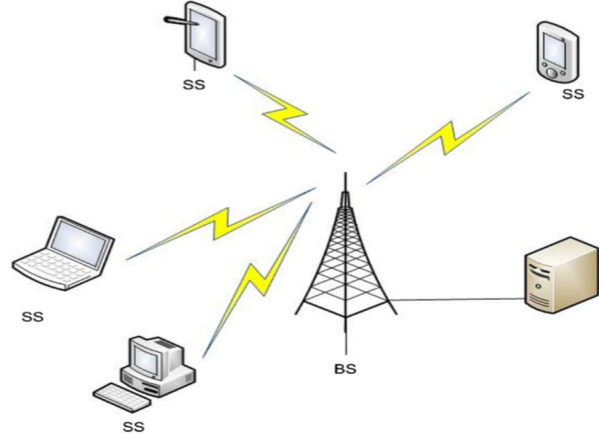

Figure 1: Simulation network topology

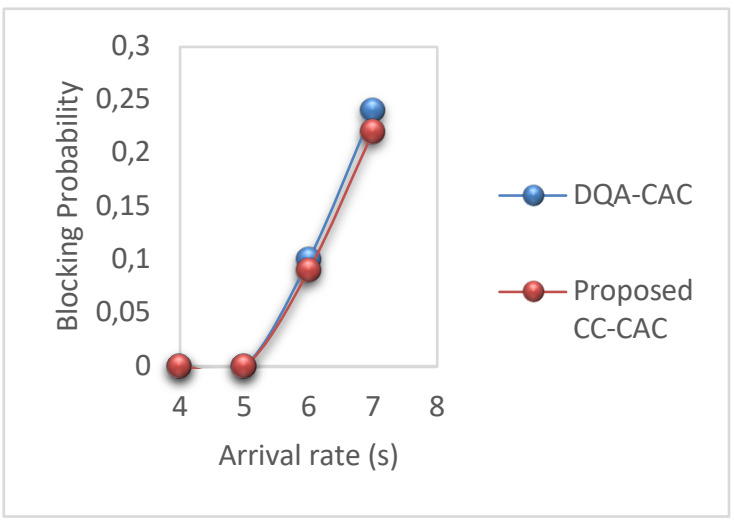

Fig. 2 Blocking Probability for light traffic

Fig. 2 shows the blocking probability when traffic is light. The proposed CC-CAC performs similarly with the existing scheme but with only slight difference. The reason for this similar performance is as a result the network has not reached the threshold operating point.

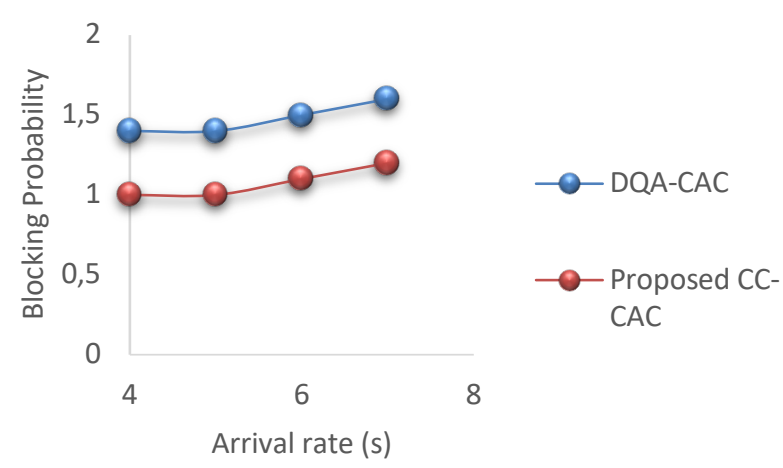

Fig. 3 Blocking Probability for Congested traffic

Fig. 3 shows blocking probability for congested traffic. The proposed performs better compared to the existing scheme. The reason for better performance is because the network is congested and our congestion mechanism is employed to minimized the traffic overflow. 


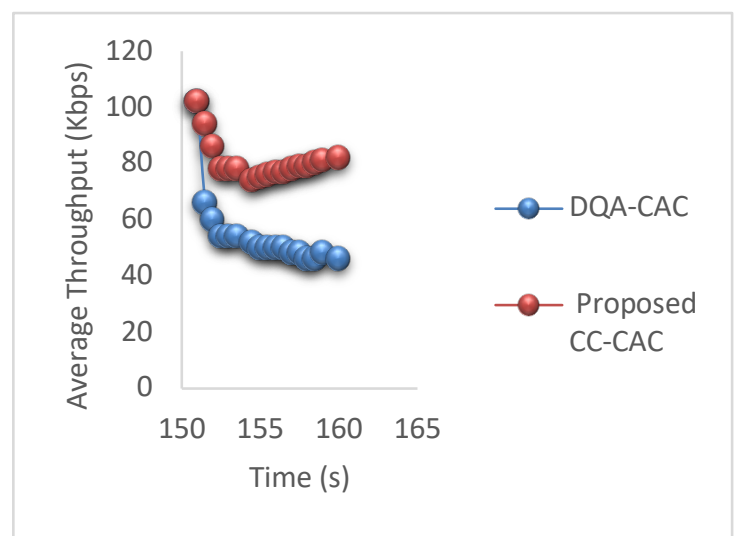

Fig. 4 Throughput for all connections

Fig.4 shows throughput for all connections. The figure shows that the proposed CC-CAC outperforms the existing scheme because of the reduction of the blocking probability.

\section{CONCLUSION}

In this paper, a $\mathrm{CC}-\mathrm{CAC}$ is proposed scheme to improve call blocking and throughput. The scheme introduces a congestion control mechanism in order to regulate the base station buffer within a certain threshold. It also deploys a dynamic bandwidth degradation mechanism to degrade varying amount of resources when resource are not sufficient to admit more connections. The proposed scheme is evaluated using extensive simulations. experiments were conducted to evaluate the performance of the The results show that the proposed CC-CAC scheme outperforms DQA-CAC in terms of reducing call blocking rates and improving throughput. In future works, we hope to incorporate a delay precheck mechanism to ensure that the delay requirements of existing and requesting delay sensitive conditions is ascertained before admission of requesting connections.

\section{ACKNOWLEDGMENT}

I would like to thank the Faculty of Engineering and Environmental Design, UDUS, Nigeria for financial support.

\section{REFERENCES}

[1]. Huan, C., Sunil, K., \& C-C. J. K. Dynamic call admission control scheme for QoS priority handoff in multimedia cellular systems" IEEE Wireless Communications and Networking Conference, 2002, 4, pp. 114-118.

[2] Kalikivayi, S., Misra, I. S., \& Saha, K. Bandwidth and delay guaranteed call admission control scheme for QOS provisioning in IEEE 802.16e mobile WiMAX. In IEEE Global Telecommunications Conference (GLOBECOM), 2008, pp. 1-6.

[3] Saidu, I., Subramaniam, S., Jaafar, A., \& Zukarnain, Z. A. “A QoS-Aware CAC with Bandwidth Reservation and Degradation Scheme in IEEE 802.16e Networks", Wireless Personal Communications 2015, 82(4), pp. 2673-2693.

[4] Lee Y, Chuah T, Loo J, Vinel A. Recent advances in radio resource management for heterogeneous LTE/LTE-A networks. IEEE Communications Surveys \& Tutorials 2014; 16(4): 2142-2180.

[5] Shu'aibu, D. S. and Syed Yusof, S. K. Link aware call admission and packet scheduling for best effort and UGS traffics in mobile WiMAX. International Journal of the Physical Sciences, 2011, 6(7), 1694-1701.

[6] Mohammed A, Solomon Y, Isah B, Saidu I. A dynamic QoS-aware call admission control algorithm for mobile broadband networks. IEEE International Conference on Computing Networking and Informatics (ICCNI) 2017; 1(1): 1-6.
[7] Romesh L., \& Iti ,S.M. A Bandwidth Efficient Adaptive Call Admission Control Scheme for QoS Provisioning in IEEE 802.16e Mobile Networks. Int J Wireless Inf Networks (2011), pp.108-116

[8] Rashidi, B.S., Taheri, H., Sabaghi. M., \& Fathi, M. A new Call Admission Control algorithm for IEEE802.16 networks", In 2010 International Conference on Computer Design and Applications (ICCDA), 2010, 4, 361-365.

[9] Wongthavarawat, K., \& Ganz, A. Packet scheduling for QoS support in IEEE 802.16 broadband wireless access systems. International Journal of Communication Systems, 2003, 16, 81-96.

[10] Wang, H., Li,W.\&Agrawal, D.P. Dynamic admission control and QoS for 802.16 wireless MAN. In Wireless Telecommunications Symposium, 2005, 60-66.

[11] Ganesh, J.S. and Bhuvaneswari, P.T.V. Enhanced Call Admission Control for WiMAX networks, In Recent Trends in Information Technology (ICRTIT), International Conference, Chennai, June 3-5, 2011, 33-36.

[12] Shwetha, D., Kumar, M.N., \& Devaraju, J.T. Modulation Aware Connection Admission Control and Uplink Scheduling Algorithm for Wimax Networks". International Journal of Wireless \& Mobile Networks, 2015, 7(1), 75-92.

[13] Wang, C., Yan, W.J. \& Lo, H.K. Dynamic admission control and bandwidth reservation for IEEE $802.16 \mathrm{e}$ mobile WiMAX networks. EURASIP Journal on Wireless Communications and Networking, 2012, $1-20$.

[14] Patel, Z. \& Dalal, U. Improved CAC Scheme for WiMAX with Adaptive Bandwidth Reservation and Degradation Policy. Int'l Journal of Computing, Communications \& instrumentation Eng. (IJCCIE), 2015, 2(2), 144-149 